\title{
Regeneração natural de um fragmento florestal de caatinga na região semi-árida do Piauí
}

\author{
Natural regeneration of a forest fragment of caatinga in the semiarid region of Piauí \\ M. M. Fernandes ${ }^{1 *}$; T. M. Oliveira ${ }^{2}$; M. R. M. Fernandes ${ }^{3}$ \\ ${ }^{1}$ Departamento de Ciências Florestais, Universidade Federal de Sergipe, 491000-000, São Cristovão-SE, Brasil \\ ${ }^{2}$ Nucleo de regularização ambiental de Ituiutaba, SEMAD, 38302-004, Ituiutaba-MG, Brasil \\ ${ }^{3}$ Programa de Pós-Graduação em Ciências Florestais, Universidade Federal do Espirito Santo, 29550-000, \\ Jeronimo Monteiro-ES, Brasil \\ *miltonmf@gmail.com
}

(Recebido em 19 de maio de 2016; aceito em 25 de dezembro de 2016)

\begin{abstract}
O trabalho teve como objetivo avaliar a estrutura fitossociológica do componente lenhoso regenerante em um fragmento florestal de caatinga no município de Gilbués - PI. No fragmento florestal de aproximadamente $30 \mathrm{ha}$, em 10 parcelas de $20 \times 50 \mathrm{~m}\left(1000 \mathrm{~m}^{2}\right)$ foram alocadas 10 sub-parcelas de $25 \mathrm{~m}^{2}$ para avaliar a regeneração natural na área. Foram amostrados os indivíduos com circunferência na altura da base $\leq 15 \mathrm{~cm}$ e nas respectivas classes de altura: 1) $0,30 \mathrm{~m} \leq \mathrm{H} \leq 1,0 \mathrm{~m}$;2) $1,1 \mathrm{~m} \leq \mathrm{H} \leq 2 \mathrm{~m}$; 3) $2,1 \mathrm{~m}$ $\leq \mathrm{H} \leq 3 \mathrm{~m}$. Baseado nos resultados obtidos, as espécies Aspidosperma cuspa, Myracroduon urundeuva, Jacaranda brasiliana, Lonchocarpus muehlbergianus, Astronium fraxinifolium e Tabebuia aurea deverão ser as espécies que irão compor o estrato arbóreo ao longo do tempo e podem ser utilizadas para o enriquecimento de reflorestamento para áreas desertificadas no nordeste do Brasil devido ao seu rápido crescimento em ambientes mais abertos.

Palavras-chave: desertificação, recuperação de áreas degradadas, fitossociologia
\end{abstract}

The study objective to evaluate the vegetation structure of woody regenerating component in a forest fragment of caatinga in the municipality of Gilbués - PI. In forest fragment of approximately $30 \mathrm{~h}$, the 10 plots of $20 \times 50 \mathrm{~m}\left(1000 \mathrm{~m}^{2}\right), 10$ sub-plots were allocated $25 \mathrm{~m}^{2}$ to evaluate the natural regeneration being sampled all individuals circumference of the base height $<15 \mathrm{~cm}$ and the following height classes: 1) 0,30 $\mathrm{m} \leq \mathrm{H} \leq 1,0 \mathrm{~m}$; 2) $1,1 \mathrm{~m} \leq \mathrm{H} \leq 2 \mathrm{~m}$; 3) $2,1 \mathrm{~m} \leq \mathrm{H} \leq 3 \mathrm{~m}$. Based on the results, the Aspidosperma cuspa, Myracroduon urundeuva, Jacaranda brasiliana, Lonchocarpus muehlbergianus, Astronium fraxinifolium and Tabebuia aurea should be the species that compose the tree layer over time and can be used for reforestation enrichment desertified areas in northeastern Brazil, due to its rapid growth in more open environments.

Keywords: Desertification, recuperation of degraded areas, Phytosociology

\section{INTRODUÇÃO}

Selecionar adequadamente as espécies florestais que irão começar o processo de sucessão ecológica em uma área degradada é uma das etapas mais críticas da restauração ecológica. Levantamentos da fitossociologia de ecossistemas florestais preservados, modificados, perturbados e degradados apresentam como objetivo não somente a seleção das espécies, mas também averiguar como utilizá-las eficientemente nos projetos de restauração ecológica [14].

Esses estudos auxiliam na definição de espécies-chave, aquelas que determinam a florística e fitossociologia do ecossistema florestal em função da sua densidade, distribuição espacial, biomassa, altura ou cobertura e que influenciam na presença das demais espécies associadas; portanto de suma importância para o sucesso da restauração ecológica em áreas degradadas [17].

Constata-se, porém, uma grande escassez de informações no que se refere à dinâmica biológica dos ecossistemas inseridos na Caatinga. A vegetação predominante no semi-árido nordestino é um complexo genericamente denominado de caatinga, cuja classificação nem sempre é fácil. Desta forma, compreender como a regeneração natural dos ecossistemas 
florestais se estabelece pode servir de subsídio para o manejo, restauração e conservação de fragmentos florestais [13].

Algumas espécies já foram testadas, como as leguminosas herbáceas (crotalárea, juncea, leucea, feijão-caupi, mamona, mисuna-preta e feijão-guandu) e gramíneas (capim buffel e sorgo granífero), para a incorporação de matéria orgânica ao solo [8]. Sinimbu (2010) [20] utilizou o consórcio das gramíneas Andropogon e Brachiaria com pinhão manso para recuperação do solo de uma área desertificada no Piauí. Entretanto, os estudos realizados para recuperação das áreas desertificadas na área baseiam-se em espécies herbáceas de ciclo curto e gramíneas exóticas que recuperam o solo, mas sem a capacidade de estabelecer um processo de sucessão ecológica.

Diante do exposto, realizou-se o presente trabalho com o objetivo de avaliar a estrutura fitossociológica do componente lenhoso regenerante em um fragmento florestal de caatinga no estágio sucessional classificado secundário inicial no município de Gilbués - PI.

\section{MATERIAL E MÉTODOS}

A área de estudo localiza-se em Gilbués - PI, no Núcleo de Pesquisa de Recuperação de Áreas Degradadas e Desertificadas (NUPERADE), com tamanho aproximado de 56 ha (951'14' S e $45^{\circ} 20^{\prime} 09^{\prime}$ 'W; Figura 1). Localizada no Sudoeste do Piauí, o município de Gilbués é considerado um núcleo de desertificação. A mineração, desmatamento e superpastejo associados a fatores edáficos e climáticos promovem e aceleram a desertificação [15]. A cobertura florestal apresenta um baixo percentual, sendo que em 2009 apresentava somente $37,07 \%$ de sua área original (18), encontrando-se fragmentada.

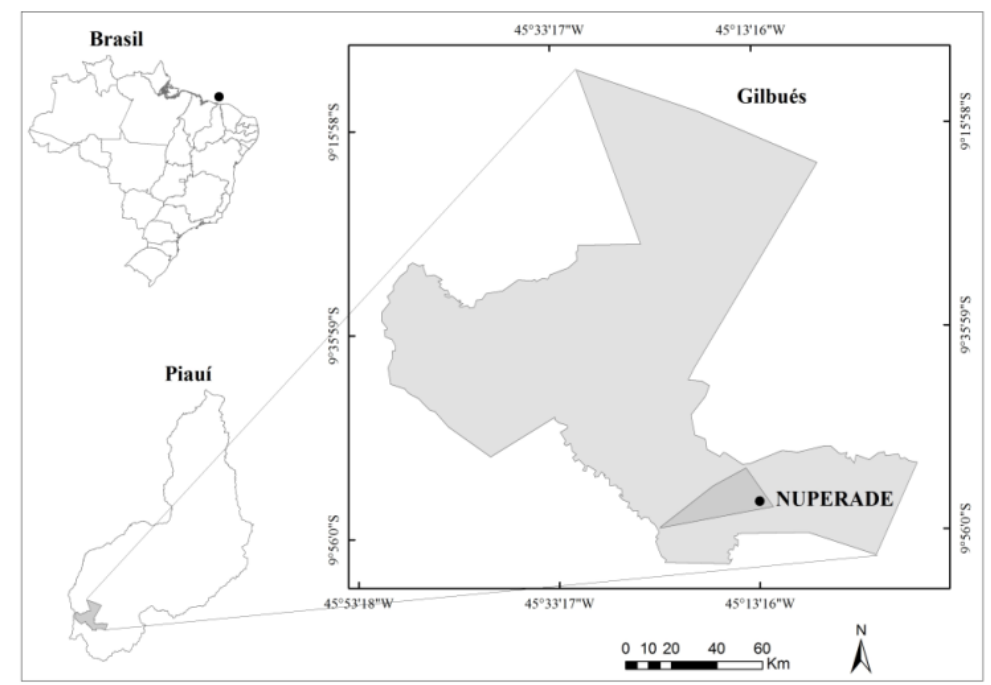

Figura 1: Localização do fragmento florestal de caatinga da área de estudo.

Segundo a classificação de Köppen, o clima da região é do tipo Aw, megatérmico, com moderada deficiência hídrica no inverno. A temperatura oscila entre a mínima de $25^{\circ} \mathrm{C}$ e máxima de $35^{\circ} \mathrm{C}$ e o mês mais frio apresenta média superior a $18^{\circ} \mathrm{C}$. A precipitação média anual é definida no regime equatorial continental, com totais variando de 800 a $1200 \mathrm{~mm}$ e apresenta pelo menos um mês com precipitação inferior a $60 \mathrm{~mm}$. O período chuvoso se estende de outubro a maio e os meses mais úmidos são de novembro a março. O solo da área de estudo foi classificado como Latossolo Vermelho - Amarelo [4].

A vegetação caracteriza-se por uma área de transição entre cerrado e caatinga [4]. Em levantamento dos indivíduos arbóreos na área de estudo por Fernandes et al. (2014) [5], o fragmento florestal encontra-se em um estágio sucessional classificado como secundário inicial, inserido em uma matriz antropizada por áreas desertificadas, sendo observadas as seguintes espécies florestais: Astronium fraxinifolium Schott, Callisthene fasciculata Mart., Crotalaria 
juncea L., Curatella americana L., Jacaranda brasiliana (Lam.) Pers., Magonia pubescens ST. Hill e Tabebuia aurea Benth. \& Hook.

Foi selecionado um fragmento florestal de aproximadamente 30 ha, sendo distribuídas 10 parcelas de $20 \times 50 \mathrm{~m}\left(1000 \mathrm{~m}^{2}\right)$ utilizadas na abordagem dos indivíduos adultos, onde foram alocadas 10 sub-parcelas de $25 \mathrm{~m}^{2}$ para avaliar a regeneração natural. Nessas parcelas foram mensuradas as alturas e diâmetros dos indivíduos regenerantes em um nível de inclusão menor ou igual a $15 \mathrm{~cm}\left(\mathrm{CAB}_{0,30 \mathrm{~m}} \leq 15 \mathrm{~cm}\right)$.

As classes de altura utilizadas foram adotadas de Souza e Soares (2013) [21], sendo que a classe I contemplou os indivíduos com $0,30 \mathrm{~m} \leq \mathrm{H} \leq 1,0 \mathrm{~m}$, classe II com altura $1,1 \mathrm{~m} \leq \mathrm{H} \leq 2,0$ $\mathrm{m}$ e classe III com altura $2,1 \mathrm{~m} \leq \mathrm{H} \leq 3,0 \mathrm{~m}$.

A identificação dos indivíduos foi realizada por meio da coleta do material botânico da regeneração natural e por comparação no Herbário Graziela Barroso da Universidade Federal do Piauí (UFPI), utilizando-se o sistema de classificação de APG III [3].

Após a coleta de dados foram calculados os parâmetros fitossociológicos da estrutura horizontal: Densidade Absoluta e Relativa, Frequência Absoluta e Relativa, Dominância Absoluta e Relativa [21]. Para a análise da estrutura vertical foi estimada a Regeneração Natural por classes de altura (RNC1, RNC2 e RNC3) utilizando a seguinte fórmula:

em que:

$$
\text { RNCi }=\text { FRi }+ \text { DRi }+\frac{\text { CRTi }}{3}
$$

$\mathrm{RNCi}$ = regeneração natural relativo da i-ésima espécie;

FR i = frequiência relativa da regeneração natural da i-ésima espécie;

DR $\mathrm{i}=$ densidade relativa da regeneração natural da i-ésima espécie;

CRT $\mathrm{i}=$ classe relativa de tamanho da regeneração da i-ésima espécie.

A RNT (Regeneração Natural Total) foi calculada de acordo com Souza e Soares (2013) [21]. Na análise da diversidade de espécies foi utilizado o Índice de Diversidade de Shannon e Equabilidade de Pielou conforme recomendado por Porto et al. (2008) [16].

\section{RESULTADOS E DISCUSSÃO}

Foram amostrados 138 indivíduos pertencentes a 12 espécies distribuídas em oito famílias botânicas. As famílias registradas e sua respectiva riqueza foram Bignoniacea (3), Fabaceae (2), Malvaceae (2), Anacardiaceae (1), Apocynaceae (1), Rubiaceae (1), Urticaceae (1) e Vochysiaceae (1). Alves Junior et al. (2013) [1] observaram na caatinga arbustivo-arbórea em Floresta, Pernambuco, que a família Fabaceae apresentou o maior número de espécies na regeneração natural, sendo esta família uma das mais numerosas em formações de Caatinga.

Quanto à distribuição do maior número de indivíduos por espécie (Figura 2), se destacaram Aspidosperma cuspa (39), Myracroduon urundeuva (32), Jacaranda brasiliana (25), Lonchocarpus muehlbergianus (13), Astronium fraxinifolium (11) e Tabebuia aurea (8).

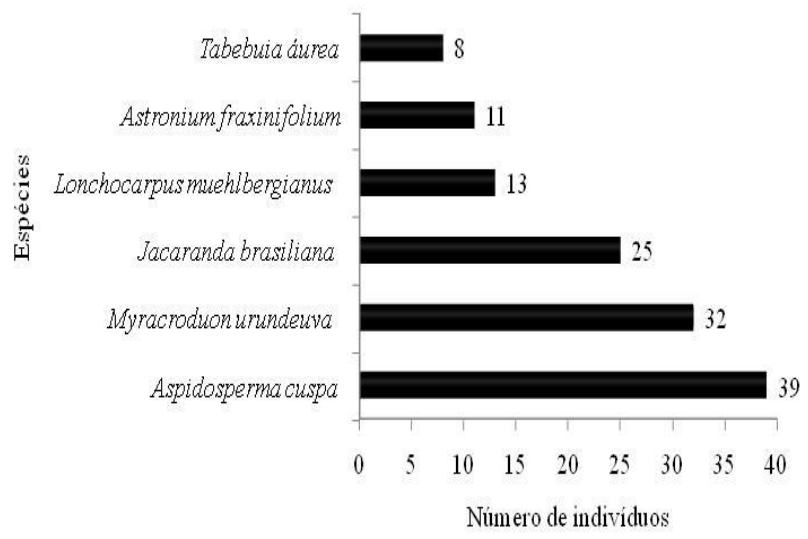

Figura 2: Espécies arbóreas com maior número de indivíduos amostrados em fragmento florestal, Gilbués - PI. 
As espécies M. urundeuva, J. brasiliana, A. fraxinifolium e $T$. aurea observados na regeneração deste estudo também foram observados em levantamento dos indivíduos arbóreos no mesmo fragmento por Fernandes et al. (2014) [5]. Desta forma, estas espécies provavelmente estão se regenerando dos indivíduos arbóreos (Tabela 1).

Observou-se que quanto à síndrome de dispersão existe um predomínio da dispersão anemocórica com sete espécies, a autocoria foi apresentada por três espécies e a zoocoria ocorreu em apenas duas espécies (Tabela 1). Marcuzzo et al. (2014) [11] observaram que duas áreas perturbadas no Rio Grande do Sul, que foram restauradas a 7 anos, apresentavam maior regeneração natural com síndrome de dispersão anemocórica e na área preservada apresentavam maior regeneração natural com dispersão zoocórica. Observa-se que as sete espécies $(58,3 \%)$ predominantes da regeneração natural do fragmento estudado apresentam dispersão anemocórica (Tabela 1). A dispersão zoocórica exerce a função da manutenção das espécies de estádios sucessionais mais avançados na sucessão ecológica e, indiretamente, no padrão de distribuição espacial e frequência das espécies florestais [7].

Tabela 1: Listagem das espécies arbóreas levantadas, os grupos ecológicos e síndrome de dispersão no fragmento florestal, Gilbués, PI.

\begin{tabular}{ccc}
\hline Espécie & GE & SD \\
\hline Myracroduon urundeuva Allemão & $\mathrm{P}$ & AUT \\
\hline Aspidosperma cuspa S.F. Blake ex Pittier & $\mathrm{P}$ & ANE \\
\hline Tabebuia aurea Benth. \& Hook. & $\mathrm{P}$ & ANE \\
\hline Jacaranda brasiliana (Lam.) Pers. & $\mathrm{P}$ & ANE \\
\hline Astronium fraxinifolium Schott. & $\mathrm{P}$ & ANE \\
\hline Lonchocarpus muehlbergianus Hassl & $\mathrm{P}$ & AUT \\
\hline Senna spectabilis (DC.) Irwin et Barn. & $\mathrm{P}$ & AUT \\
\hline Luehea grandiflora Mart. \& Zucc. & $\mathrm{P}$ & ANE \\
\hline Pseudobombax grandiflorum & $\mathrm{P}$ & ANE \\
\hline Alibertia edulis (L. Rich.) A. Rich & $\mathrm{P}$ & ZOO \\
\hline Cecropia sciadophylla Mart. & $\mathrm{P}$ & ZOO \\
\hline Callisthene fasciculata Mart. & $\mathrm{NP}$ & ANE
\end{tabular}

GE: Grupo ecológico; P(Pioneira, Secundária Inicial) e NP: Não Pioneira; SD: Síndrome de Dispersão. AUT: Autocórica; ANE: Anemórica e ZOO: Zoocórica.

As espécies A. cuspa e L. muehlbergianus embora não estivessem presentes entre as espécies arbóreas adultas, apresentam indivíduos regenerantes. Estas espécies apresentam dispersão anemocórica (Tabela 1), o que favorece a dispersão de longas distâncias, inclusive de fragmentos próximos da área. Aparício et al. (2014) [2] avaliando a regeneração natural na Floresta Amazônica no Amapá também observaram que algumas espécies com dispersão anemocórica, presentes na regeneração natural, não apresentavam indivíduos no estrato arbóreo.

Praticamente todas as espécies são classificadas como pioneiras, com exceção de Callisthene fasciculata (Tabela 1). A área estudada está inserida numa matriz antrópica de áreas em processo de desertificação, o que favorece um maior efeito de borda, sendo que as espécies pioneiras são mais adaptadas a maior incidência de luz.

As espécies A. cuspa, M. urundeuva, J. brasiliana, L. muehlbergianus, A. fraxinifolium e $T$. aurea apresentaram os maiores valores de densidade relativa $(92,76 \%)$ e corresponderam a $92,70 \%$ do total dos indivíduos presentes. A densidade total e a dominância absoluta foram estimadas em 172,1 ind.ha ${ }^{-1}$ e $0,28 \mathrm{~m}^{2} . \mathrm{ha}^{-1}$, respectivamente (Tabela 2). A densidade total neste estudo é baixa quando comparada a Marcuzzo et al. (2014) [11] que observaram uma densidade da regeneração natural de 11.388 ind.ha $^{-1} \mathrm{em}$ uma área perturbada reflorestada a 7 anos na Mata Atlântica no Sul do Brasil. Silva et al. (2012) [19], avaliando a regeneração natural de uma caatinga antropizada a 30 anos, observaram uma densidade de 8.116 ind.ha $^{-1}$. 
Tabela 2: Parâmetros da estrutura da regeneração natural amostrada no fragmento florestal, Gilbués -

\begin{tabular}{cccccccccc}
\hline Espécie & DA & DR & DoA & DoR & VI & RNC $_{1}$ & RNC $_{2}$ & RNC $_{3}$ & RNT \\
\hline $\begin{array}{c}\text { Myracroduon urundeuva } \\
\text { Allemão }\end{array}$ & 40,0 & 23,19 & 0,017 & 20,10 & 61,06 & 27,68 & 24,22 & 24,03 & 25,31 \\
\hline $\begin{array}{c}\text { Aspidosperma cuspa S.F. Blake } \\
\text { ex Pittier }\end{array}$ & 48,7 & 28,26 & 0,034 & 10,19 & 56,23 & 34,82 & 30,47 & 30,23 & 31,84 \\
\hline Tabebuia aurea Benth. \& Hook. & 10,0 & 5,80 & 0,050 & 15,18 & 34,31 & 5,36 & 4,69 & 4,65 & 4,90 \\
\hline $\begin{array}{c}\text { Jacaranda brasiliana } \text { (Lam.) } \\
\text { Pers. }\end{array}$ & 31,2 & 18,12 & 0,064 & 19,40 & 50,85 & 20,54 & 17,97 & 17,83 & 18,78 \\
\hline $\begin{array}{c}\text { Astronium fraxinifolium Schott. } \\
\text { Lonchocarpus muehlbergianus } \\
\text { Hassl }\end{array}$ & 13,7 & 7,97 & 0,058 & 17,57 & 34,43 & 0,00 & 6,25 & 6,20 & 4,15 \\
\hline $\begin{array}{c}\text { Senna spectabilis } \text { (DC.) Irwin et } \\
\text { Barn. }\end{array}$ & 1,2 & 0,72 & 0,000 & 0,13 & 3,08 & 0,00 & 0,78 & 0,78 & 0,52 \\
\hline $\begin{array}{c}\text { Luehea grandiflora } \text { Mart. \& } \\
\text { Zucc. }\end{array}$ & 2,5 & 1,45 & 0,017 & 5,25 & 8,92 & 0,00 & 0,78 & 0,78 & 0,52 \\
\hline Pseudobombax grandiflorum & 2,5 & 1,45 & 0,003 & 0,89 & 6,79 & 0,00 & 1,56 & 1,55 & 1,04 \\
\hline $\begin{array}{c}\text { Alibertia edulis } \text { (L. Rich.) A. } \\
\text { Rich }\end{array}$ & 1,2 & 0,72 & 0,000 & 0,01 & 2,95 & 0,00 & 0,78 & 0,78 & 0,52 \\
\hline Cecropia sciadophylla Mart. & 1,2 & 0,72 & 0,005 & 1,53 & 4,48 & 0,00 & 0,00 & 0,78 & 0,26 \\
\hline Callisthene fasciculata Mart. & 3,7 & 2,18 & 0,024 & 7,42 & 11,82 & 0,00 & 2,34 & 2,31 & 1,54 \\
\hline Total & 172,1 & 100 & 0,28 & 100 & 300 & 100 & 100 & 100 & 100 \\
\hline
\end{tabular}

DA: densidade absoluta (ind.ha ${ }^{-1}$ ); DR: densidade relativa (\%); DoA: dominância absoluta $\left(m^{2} . h a^{-1}\right) ;$ DoR: dominância relativa (\%); VI: valor de importância; $R N C_{1}$ : Regeneração Natural da Classe de altura 1 (\%); $R N C_{2}$ : Regeneração Natural da Classe de altura 2 (\%); RNC 3 : Regeneração Natural da Classe de altura $3(\%)$; RNT: Regeneração Natural Total (\%).

Aspidosperma cuspa, Myracroduon urundeuva, Jacaranda brasiliana e Lonchocarpus muehlbergianus foram as que mais se destacaram correspondendo a 94,65\% da $\mathrm{RNC}_{1} ; \mathrm{RNC}_{2}$ com 82,58 \%; $\mathrm{RNC}_{3} \operatorname{com} 82,17 \%$ e na RNT com 86,55 \% (Tabela 2 e Figura 4). A espécie $A$. cuspa apresentou maior percentual nas três classes de altura e da regeneração natural total (RNT). De acordo com Fernandes et al. (2014) [5], o solo apresenta uma boa fertilidade com altas concentrações de cálcio, magnésio, potássio e fósforo que favorecem a regeneração natural dessa espécie. Menino (2009) [12], avaliando a florística e fitossociologia de uma área de transição entre caatinga e cerrado no Norte de Minas Gerais, observou que a regeneração de $A$. cuspa estava associada à presença de solos férteis conforme observado neste estudo.

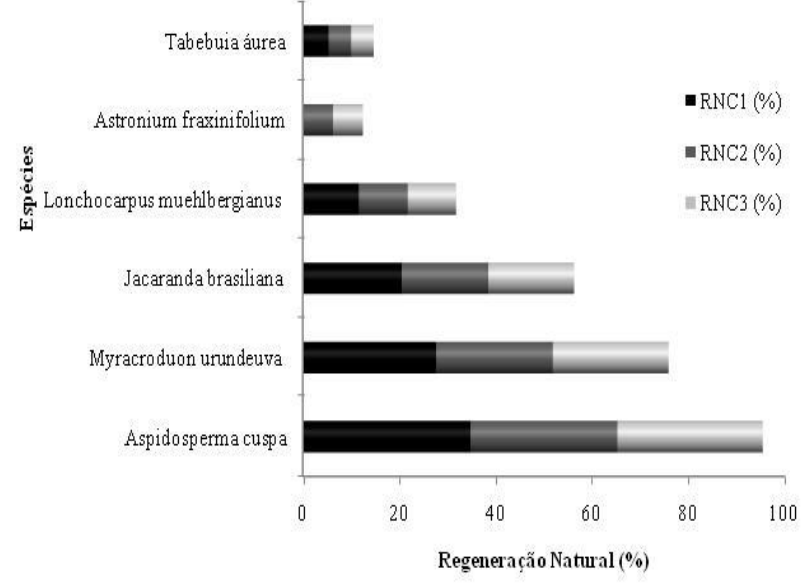

Figura 4: Espécies arbóreas que apresentaram maiores índices de Regeneração Natural Total (RNT) da população amostrada, expressos em porcentagem, no fragmento florestal em Gilbués - PI.

As espécies A. cuspa, M. urundeuva, J. brasiliana, L. muehlbergianus e T. aurea apresentaram uma distribuição equilibrada nas classes de altura da regeneração natural total CI, CII e CIII. Desta forma, observa-se que não existe o predomínio de uma determinada classe de altura da regeneração natural total. Os maiores índices de regeneração natural total ocorreram nas mesmas espécies que obtiveram maiores valores de importância (Figura 4 e Tabela 2). Este 
comportamento foi observado por Lima et al. (2013) [10] na regeneração natural de uma floresta ombrófila densa em Pernambuco, onde as espécies que apresentaram maiores índices de regeneração total foram as que obtiveram maiores valores de importância.

Myracroduon urundeuva apresentou o segundo maior valor de importância, apresentando uma boa regeneração nas diferentes classes de altura e na regeneração natural total, estando bem estabelecida na área desertificada. Este comportamento foi observado por Ferraz et al. (2014) [6] em uma caatinga conservada, onde essa espécie foi a mais competitiva substituindo gradativamente espécies iniciais, com elevada capacidade de dispersão e estabelecimento.

$\mathrm{O}$ valor do índice de diversidade de Shannon (H') obtido para a regeneração natural da área de estudo foi de 1,90 nats. ind ${ }^{-1}$ e o índice de equabilidade de Pielou foi de 0,76. Kunz e Martins (2014) [9] observaram uma baixa diversidade florística em uma floresta em estágio médio de sucessão de 1,69 nats. ind ${ }^{-1}$ próximo ao observado neste estudo, decorrente da alta abundância de poucas espécies. A equabilidade de Pielou observada neste estudo foi similar ao observado por Alves Junior et al. (2013) [1] trabalhando em uma caatinga arbórea - arbustiva no semiárido de Pernambuco, que observaram uma equabilidade de 0,70 .

\section{CONCLUSÃO}

Baseado nos resultados obtidos, as espécies Aspidosperma cuspa, Myracroduon urundeuva, Jacaranda brasiliana, Lonchocarpus muehlbergianus, Astronium fraxinifolium e Tabebuia aurea deverão ser as espécies que irão compor o estrato arbóreo ao longo do tempo e podem ser utilizadas para o enriquecimento de reflorestamento para áreas desertificadas no nordeste do Brasil devido ao seu rápido crescimento em ambientes mais abertos.

\section{REFERÊNCIAS BIBLIOGRÁFICAS}

1. Alves Junior FT, Ferreira RLC, Silva JAA, Marangon LC, Cespedes GHG. Regeneração natural de uma área de caatinga no Sertão Pernambucano, Nordeste do Brasil. Cerne 2013;19(2):229-235, doi.org/10.1590/S0104-77602013000200006.

2. Aparicio, PS, Sotta ED, Guedes MC, Aparicio WCS, Oliveira LP, Souza RN. Níveis de regeneração natural em floresta de terra firme no Amapá - Brasil. Rev Árvore 2014;38(4):699710, doi.org/10.1590/S0100-67622014000400013.

3. III - APG Angiosperm Phylogeny Group III. An update of the Angiosperm Phylogeny Group classification for the orders and families of flowering plants. Bot J Linnean Soc 2009;161(2):105-121.

4. Fernandes MM, Silva MD, Veloso MEC, Oliveira TM, Fernandes MRM, Sampaio FMT. Biomassa microbiana e matéria orgânica em áreas desertificadas revegetadas com pinhão-manso solteiro e consorciado com gramínea no Sul do Piauí. Agrária 2013;8(3):464-469, doi:10.5039/agraria.v8i3a2392.

5. Fernandes MM, Oliveira TM, Fernandes MRM, Castro VC, Alves AR. Aspectos biológicos e espécies potenciais para restauração ecológica de áreas em desertificação no Sul do Piauí - Brasil. Rev Verde 2014;9(2):6-13.

6. Ferraz JS, Ferreira RLC, Silva JA, Meunier IMJ, Santos MVF. Estrutura do componente arbustivoarbóreo da vegetação em duas áreas de caatinga, no município de Floresta, Pernambuco. Rev Árvore 2014;38(6):1055-1064, doi.org/10.1590/S0100-67622014000600010.

7. Franco KS, Martins SV, Faria PCL, Ribeiro GA, Miranda Neto A. Estrato de regeneração natural de um trecho de Floresta Estacional Semidecidual, Viçosa. Rev Árvore 2014;38(1):31-40, doi.org/10.1590/S0100-67622014000100003.

8. Fundação de Amparo à Pesquisa do Estado do Piauí - FAPEPI. Relatório sobre a desertificação no Sul do Piauí. Rev Sapiência 2006. Teresina: FAPEPI, n.7.

9. Kunz SH, Martins SV. Regeneração natural de floresta estacional semidecidual em diferentes estágios sucessionais (Zona da Mata, MG, Brasil). Floresta 2014;44(1):111-124, doi.org/10.5380/rf.v44il.30736.

10. Lima AS, Feliciano ALP, Marangon LC, Oliveira LSB, Pessoa MML. Regeneração natural de um fragmento de Floresta Ombrófila Densa na Bacia Hidrográfica do Rio Capibaribe, PE. Agrária 2013;8(2):273-278, doi:10.5039/agraria.v8i2a2369. 
11. Marcuzzo SB, Araújo MM, Rorato DG, Machado JM. Comparação entre áreas em restauração e área de referência no Rio Grande do Sul, Brasil. Rev Árvore 2014;38(6):961-972, doi.org/10.1590/S010067622014000600001

12. Menino GCO. Florística e estrutura da regeneração natural da vegetação ciliar do rio Pandeiros, norte de Minas Gerais. [Dissertação] Montes Claros: Universidade Estadual de Montes Claros; 2009. 120 p.

13. Narvaes IS, Longhi S, Brena D. Florística e classificação da regeneração natural em Floresta Ombrófila Mista na Floresta Nacional de São Francisco de Paula, RS. Ciência Florestal 2008;18(2):233-245, doi.org/10.5902/19805098460.

14. Neri AV, Soares MP, Meira JAAN, Dias LE. Espécies de cerrado com potencial para recuperação de áreas degradadas por mineração de ouro, Paracatu - MG. Rev Árvore 2011;35(4):907-918.

15. Patrício MCM, Silva VMA, Ramos ARD. Gilbués - núcleo de desertificação do Piauí, caracterização física, variabilidade climática e impactos ambientais. Rev Polêmica 2012;11(3):23 - 30.

16. Porto ML, Assunção AF, Girardi-Deiro AM, Forneck ED, Zocche JJ, Pfadenhauer JS, Bortolotti JS, Oliveira MLAA, Wildi O, Termignoni RR, Frizzo TCE. Comunidades vegetais e fitossociologia: fundamentos para avaliação e manejo de ecossistemas. Porto Alegre - RS: Editora UFRGS; 2008. 240 p.

17. Salomão RP, Brienza Júnior S, Santana AC. Análise da florística e estrutura de floresta primária visando à seleção de espécies-chave, através de análise multivariada, para a restauração de áreas mineradas em Unidades de Conservação. Rev Árvore 2012;36(6):989-1007, doi.org/10.1590/S010067622012000600001 .

18. Silva IAS. Conflitos territoriais em Gilbués - PI: desenvolvimento do agronégocio e degradação ambiental. Rev Tocantinense Geogr, 2016;5(8):29-52, doi.org/10.22241/2317-9430/rtg.v5n8p29-52.

19. Silva SO, Ferreira RLC, Alves JRTF, Lira MA. Regeneração natural em um remanescente de Caatinga com diferentes históricos de uso no agreste Pernambucano. Rev Árvore 2012;36(3):441-450.

20. Sinibum F. Tecnologias para frear a erosão. Série Documentos da Embrapa Meio Norte, Teresina, 2010;5(953):12-20.

21. Souza AL, Soares CPB. Florestas Nativas: estrutura, dinâmica e manejo. Viçosa, MG: Ed. UFV; 2013. 322 p. 